\title{
La construction politique d'un sujet « afro » ou « noir » au Mexique
}

The Political Construction of "the Afro" and "the Black" in Mexico

La construcción política de «lo afro» $y$ «lo negro» en México

\section{Gloria Lara}

Traducteur : Solange Lebourges

\section{(2) OpenEdition Journals}

\section{Édition électronique}

URL : https://journals.openedition.org/remi/5348

DOI : $10.4000 /$ remi.5348

ISSN : $1777-5418$

Éditeur

Université de Poitiers

Édition imprimée

Date de publication : 1 juin 2011

Pagination : 89-106

ISBN : 979-10-90426-00-9

ISSN : 0765-0752

\section{Référence électronique}

Gloria Lara, «La construction politique d'un sujet « afro » ou « noir » au Mexique », Revue européenne des migrations internationales [En ligne], vol. $27-n^{\circ} 1$ | 2011, mis en ligne le 01 juin 2014, consulté le 15 avril 2022. URL : http://journals.openedition.org/remi/5348 ; DOI : https://doi.org/10.4000/remi.5348 


\title{
La construction politique d'un sujet « afro » ou « noir » au Mexique
}

\author{
Gloria LARA $^{1}$
}

\section{INTRODUCTION ${ }^{2}$}

epuis un peu plus de cinq ans, la « question afrodescendante » est à l'ordre
du jour des sciences sociales au Mexique. L'intérêt pour ce sujet a été dans une grande mesure motivé par la revendication explicite, par certains leaders et certaines organisations, d'une identité « afromexicaine » ou « noire ». Plus globalement, depuis les années 1980, le processus de construction du discours ethnique se nourrit des interventions des intellectuels nationaux et étrangers ainsi que des organisations du mouvement noir sous d'autres latitudes. Cette construction d'une identité ethnico-raciale « nouvelle » pour le Mexique, est problématique pour ceux qui la revendiquent et qui, sans être très nombreux, constituent cependant une nébuleuse gravitant autour de quelques noyaux ou groupes organisés. On observe au sein de ce courant des logiques diverses, et par moment divergentes, à partir desquelles chaque groupe intéressé définit cette identité. Ainsi certains leaders utilisent-ils pour s'auto-définir des catégories raciales tandis que d'autres formulent des stratégies pour échapper à ce qu'ils considèrent être une stigmatisation. À cette variété d'options viennent s'ajouter des conflits ou des mises en concurrence entre les groupes qui participent au débat et affichent leurs appartenances à d'autres réseaux et d'autres mouvements dans lesquels ils sont insérés, et qui ont eux-mêmes, de leurs côtés, des canaux d'interlocution diversifiés (partis politiques, syndicats, ONG, églises).

La construction de catégories ethniques ou raciales dans les milieux intellectuels passe, elle aussi, par des chemins sinueux, surtout en anthropologie. Un des débats

1 Anthropologue, Universidad Michoacana de San Nicolas Hidalgo, Facultad de Historia, Ciudad Universitaria. Av. Universidad No. 1600, Col. Villa Universidad, C.P. 58060, Tel/ Fax (443) 3270003 /3164177, Morelia, Michoacán, México ; laram.gloria@gmail.com L'article a été traduit de l'espagnol au français par Solange Lebourges.

2 Je remercie l'appui d'Afrodesc (ANR) et de Eurescl (programme européen 7ème PCRD), programmes grâce auxquels la réalisation du travail de terrain a été possible, et particulièrement Elisabeth Cunin et Odile Hoffmann. 
centraux a porté sur le besoin, exprimé par certains intellectuels, de définir l'existence d' " une culture noire ». Cette démarche se fonde sur une conception de la diversité culturelle comprise comme un ensemble de contenus, une conception qui présente la « différence culturelle » comme un fait primordial. Cette approche de la culture s'appuie sur des catégories raciales élaborées et utilisées par les acteurs sociaux puis essentialisées, comme si elles correspondaient à des attributs biologiques immuables s'exprimant dans des traits culturels objectivables (danses, musique, tradition de l'oralité). Ce discours universitaire, en accord avec le discours multiculturaliste institutionnel, est celui qui s'est diffusé le plus largement parmi les militants et les organisations qui demandent la reconnaissance des « peuples noirs » dans le pays et la création de politiques publiques en faveur de ces populations. Comme dans d'autres contextes américains, "la différence » est posée comme un fait social et politique majeur et fondateur, qui fait référence à l'histoire de la conquête et de la colonisation qui mit face à face des groupes d'origines diverses, porteurs d'expériences distinctes (pour faire court, l'Europe et la Méso-Amérique). Cette notion de « différence » a été actualisée et légitimée dans les arènes nationales et internationales par les réflexions philosophiques et les mesures politiques adoptées en faveur de la « reconnaissance de la différence » (Kymlicka 1996 ; Taylor 1994).

Les interprétations essentialistes sont fortement critiquables dans la mesure où elles reposent sur une naturalisation de " la différence », sur la base de traits physiques « culturellement distinctifs », qui tend à organiser la diversité en termes raciaux, dans un contexte où ce mode de catégorisations légitime des processus de domination/subordination.

Pour autant, il nous paraît important de reconnaître l'existence d'une population afrodescendante dans le pays, tout comme celle des luttes politiques menées pour sa reconnaissance. Nous assistons probablement à des processus d'ethnogenèse élaborés autour de conflits et de luttes menés par des personnes et des groupes qui cherchent ainsi à se repositionner au sein de, et contre une histoire générale de domination (Hill, 1996). Les groupes intéressés (ici les groupes noirs ou afrodescendants) peuvent aussi proposer la redéfinition de catégories anciennes (en réintroduisant « la race » par exemple) à la lumière d'une nouvelle situation politique (Bilby, 1996). Face aux mobilisations observées, il est nécessaire de distinguer constamment entre les niveaux d'organisation selon qu'ils proviennent de l'intérieur (emic) ou de l'extérieur (etic) des collectifs sociaux étudiés. Ceci permet de mieux comprendre les logiques sur lesquelles ces groupes fondent leurs formes d'expression identitaire et culturelle. Par ailleurs, il faut se rappeler que la capacité de négociation (et d'élaboration de discours) des acteurs de base est toujours contrainte par les rapports sociaux de domination et de pouvoir qui sous-tendent les situations concrètes.

En m'appuyant sur des travaux antérieurs, cet article s'attache à décrire l'action politique des organisations autoproclamées " afrodescendantes » au Mexique et les processus d'ethnicisation nourris par la mise en saillance de « traits culturels » et phénotypiques. Une première partie situe l'émergence du sujet dans les études universitaires et l'intervention des institutions de l'État ; la seconde montre quels sont les protagonistes de l'action ethno-politique. La conclusion souligne les catégories de « la différence » qui sont au cœur du débat concernant la revendication de la population afrodescendante au Mexique. 


\section{L'ÉMERGENCE DU SUJET DU « NOIR » DANS LES MILIEUX UNIVERSITAIRES ET LES INSTITUTIONS GOUVERNEMEN- TALES}

J'essaierai tout d'abord de cerner les populations afrodescendantes contemporaines au Mexique, tout en précisant d'entrée qu'il est problématique de définir ceux qui composent cette population. Jusqu'à aujourd'hui, cette dernière n'est pas représentée dans l'imaginaire national « officiel » (celui qui est véhiculé par les médias et le système d'enseignement nationaux). Il n'existe pas dans le pays de catégorisation instituée ou consensuelle à ce propos, ni de la part des institutions gouvernementales ni de celle de la société civile ou des milieux universitaires. La population en question - celle qui aujourd'hui tend à se reconnaître comme " noire » - est caractérisée par une forte hétérogénéité, tant sur le plan de sa situation géographique (urbaine et rurale, dans de petites localités comme dans les métropoles, dans toutes les régions du pays) que de ses formes d'insertion dans la société globale et de ses relations avec d'autres groupes sociaux.

D'un point de vue juridique, la constitution politique mexicaine reconnaît une composition pluriculturelle de la nation, reposant à l'origine sur les populations indigènes. Dans la législation fédérale, la population afrodescendante n'est pas mentionnée comme élément de la mosaïque culturelle. Seule une loi régionale de 1998 reconnaît cette population, appelée groupe ethnique « afromexicain $»^{3}$, dans l'État d'Oaxaca. Il faut souligner qu'il n'existe pas de démarcation de territoires spécifiques associés à ces populations, et qu'il n'y a pas eu non plus d'attributions spécifiques de titres de propriété foncière selon un statut ethnique, comme c'est parfois le cas pour les populations indiennes de ce même État.

Depuis 2005 environ, les débats autour de la reconnaissance et des droits des populations afrodescendantes au Mexique se développent autour d'un argumentaire racialisé et ethnicisé. Le sujet comporte toutefois d'autres dimensions, en particulier l'exigence de l'exercice de la citoyenneté liée à l'égalité des droits, à l'accès aux programmes gouvernementaux et à la participation politique. Ces débats doivent être replacés dans le contexte des conditions locales et nationales de mobilisation citoyenne préexistantes, ainsi que des discours qui émanent d'organisations et de réseaux d'organisation noirs, des milieux académiques, des agences internationales ou des déclarations internationales comme celle de Durban, entre autres. Ce contexte constitue un terreau favorable pour l'action politique des leaders et des organisations revendiquant les droits des populations afrodescendantes au Mexique, en même temps qu'une source d'inspiration pour les dirigeants à la recherche d'instruments légitimes de catégorisation.

Les organisations militantes les plus actives ont peu à peu élaboré leurs conceptions du « noir » ou de l' " afro », en les nourrissant de discours et d'outils juridiques repris au mouvement indigène au $\mathrm{Mexique}^{4}$, des multiples contacts qu'ils ont noués avec

3 Voir Ley de comunidades y Pueblos Indígenas (Loi des Communautés et Peuples Indigènes) de l'État d'Oaxaca, 1998.

4 En particulier des cadres juridiques qui légitiment leurs revendications identitaires, comme la convention 169 de l'Organisation Internationale du Travail. 
des organisations afrolatines et afroaméricaines, ainsi que des réflexions d'intellectuels nationaux et étrangers qui soutiennent la reconnaissance d'une " culture noire » dans le pays. Ainsi, la visibilité de ces organisations s'est-elle déployée grâce aux alliances qu'elles ont établies avec divers acteurs et selon des formes d'expression diversifiées, allant des discours universitaires jusqu'aux déclarations internationales.

Au Mexique, le précurseur des études sur les afrodescendants fut Gonzalo Aguirre Beltrán. Ses travaux de type ethno-historique ont débuté dans les années 1940 ; il y soulignait les apports des populations d'origine africaine à la culture et à l'histoire mexicaine, tout en situant les différences ethniques dans des relations de pouvoir. Cependant, en même temps, ce chercheur et fonctionnaire gouvernemental pronostiquait la prochaine disparition de la population " afrométisse $»^{5}$ en tant que groupe social spécifique et son intégration à la population métisse. Le métissage était alors conçu comme l'intégration des racines biologiques et culturelles, intégration censée unifier la nation. Aguirre Beltrán, qui fut l'un des principaux théoriciens de l'intégrationnisme, pensait que l'unification nationale apporterait une certaine homogénéisation quant à la composition ethnique, économique, sociale et politique du pays. En conséquence, il envisageait la rapide fusion de la population afrométisse dans l'ensemble de la société et sa disparition en tant que telle, au motif que les « traits culturels » pouvant soutenir une identification ethnique « noire » étaient peu nombreux et que les traits phénotypiques tendraient à disparaître au fil du temps, avec le métissage entre les populations (Aguirre Beltrán, 1984 [1946]).

L'extinction annoncée du groupe social afrométis a conduit à l'extinction des études anthropologiques sur ce thème (Hoffmann, 2006) et les quelques productions qui ont suivi celles d'Aguirre Beltrán ne se sont intéressées aux populations afrométisses que dans la mesure où elles étaient en interaction avec les populations indigènes, considérées comme les seuls " vrais » sujets ethniques. Au milieu des années 1980, le thème des afrodescendants s'est toutefois renouvelé grâce à une institution gouvernementale : la Dirección General de Culturas Populares (Direction Générale des Cultures Populaires). Au sein de cette institution, l'anthropologue Guillermo Bonfil créa le Programme de « La tercera raíz » (La troisième racine), dont l'objectif était la reconnaissance des apports culturels des populations d'origine africaine au Mexique. Dans ce programme furent déployées une série d'actions destinées à promouvoir la musique, la danse et les traditions orales dans les régions du pays où leurs apports se distinguaient particulièrement. Ainsi furent promues des expressions culturelles faisant partie d'un ensemble de pratiques régionales qui, par ailleurs à l'époque et dans les régions considérées, n'étaient pas forcément caractérisées comme « afrométisses ». Plusieurs groupes locaux, principalement dans les États de Veracruz, du Guerrero et d'Oaxaca, furent favorisés par ce programme. Dans le cas des groupes culturels militants du Guerrero et d'Oaxaca, le programme favorisa les actions destinées à « récupérer » et « sauvegarder » l'histoire de l'esclavage et à la rendre explicite en tant que partie de leur histoire collective. Cette action institutionnelle a permis des publications, des productions discographiques (de corridos, de poèmes traditionnels et de sones), la diffusion de danses afrométisses en dehors de leur région d'origine, et la création dans l'État du Guerrero, de la Casa de la Cultura (Maison de la

5 Le terme afrométis est utilisé par Aguirre Beltrán pour signaler l'hybridation du « facteur noir » prépondérant avec d'autres groupes sociaux (voir Aguirre Beltrán, 1984 [1946]).

REMI 2011 (27) 1 pp. 89-106 
Culture) au village de San Nicolás Tolentino et du Museo de las Culturas Afromestizas de Cuajinicuilapa (Musée des Cultures Afrométisses de Cuajinicuilapa). Le soutien du Programme Tercera Raíz aux actions locales diminua par la suite, jusqu'à disparaître avec le programme lui-même en 2001.

Actuellement, l'institution gouvernementale en charge de ces thèmes est le Consejo Nacional para Prevenir la Discriminación (CONAPRED) (Conseil National pour la Prévention de la Discrimination). Cet organisme a été créé en 2003 par l'État pour « promouvoir des politiques et des mesures tendant à contribuer au développement culturel et social, à avancer vers l'inclusion sociale et à garantir le droit à l'égalité $»^{6}$. La prise en charge des thématiques " afro » par le CONAPRED est en grande partie liée à la participation du gouvernement mexicain à des initiatives internationales autour de la mémoire de l'esclavage et de la diaspora africaine, ainsi qu'à son adhésion et sa collaboration à des actions contre la discrimination ${ }^{7}$. En témoigne le rapport que le Comité des Nations Unies pour l'élimination de la discrimination raciale a demandé au gouvernement mexicain au sujet des actions qu'il promeut pour protéger les droits de l'homme des communautés afrodescendantes dans le pays (SRE, 2007a et 2007b) ${ }^{8}$.

Les premières initiatives du CONAPRED sur le thème des afrodescendants ont été le financement d'études et de rapports. Depuis 2006, deux études ont été réalisées et une autre est en cours. Les deux premières avaient pour but de rendre compte des conditions de vie des populations afrodescendantes, des processus différenciés de construction identitaire et des phénomènes de discrimination. Ces études localisent la population afrodescendante principalement dans les États d'Oaxaca et du Guerrero mais aussi dans le nord du pays (Flores, 2006 ; 2007) et recensent les organisations et les leaders qui lui sont liés. En 2008, le CONAPRED commença à se rapprocher des dirigeants des organisations et à se poser comme interlocuteur de l'État pour relayer les demandes de certaines d'entre elles. À ce titre, le CONAPRED a mené des discussions inter-institutionnelles, surtout avec l'Instituto Nacional de Estadística Geografía e Informática (INEGI) (Institut National des Statistiques, de la Géographie et de l'Informatique), auquel il a demandé

6 Diario Oficial de la Federación (Journal Officiel de la Fédération), 11 juin 2003. Ses fonctions comprennent la réception et le traitement des réclamations et des plaintes pour actes discriminatoires commis par des particuliers ou les pouvoirs publics.

7 Comme le projet de la Route de l'Esclave de l'UNESCO et les Conférences Mondiales sur le Racisme organisées à Durban en 2001 et à Brasilia en 2006. Parmi les propositions que le gouvernement mexicain soutient, on remarque « l'établissement de mécanismes de promotion des droits de l'enfance et de l'adolescence des afrodescendants dans les Amériques et l'adoption de programmes de formation aux droits de l'homme pour les agents des systèmes judiciaire, pénitentiaire et policier, considérant les effets pernicieux de la discrimination en ce qui concerne l'application de la justice, spécialement sur la jeunesse afrodescendante de la région » (Nations Unies, 2007).

8 « Le Comité recommande à l'État concerné de fournir des informations au sujet des communautés afrodescendantes qui sont numériquement petites et plus vulnérables et qui pour cela doivent compter sur toutes les garanties de protection que la Convention établit », Comité para la Eliminación de la Discriminación Racial (Comité pour l'Élimination de la Discrimination Raciale), en réponse à la requête exprimée dans le paragraphe 21 de ses " Observaciones Finales para México » émises en 2006 (Nations Unies, 2006). 
d'inclure des questions dans le recensement de population de 2010 afin de dénombrer la population afrodescendante. Il a pour cela réuni des responsables associatifs, des universitaires et des représentants de différentes instances gouvernementales intéressées par le thème ethnique (essentiellement des gens travaillant en milieu indien) afin d'élaborer un agenda des besoins et des demandes de ces populations. Au cours de ces réunions, il est apparu que la préoccupation centrale des organisations de terrain était la mise en œuvre de politiques publiques d'assistance au bénéfice des populations afrodescendantes. La grande question devint alors, pour les institutions et les fonctionnaires en charge de ces politiques d'identifier les bénéficiaires potentiels : comment définir les sujets de droits spécifiques, les porteurs d'une identité culturelle particulière ? Les organisations et certains de leurs représentants pensent que le recensement est un moyen, en fait le seul, permettant de répondre à ces questions. Cette proposition pose de multiples problèmes et sa pertinence même est discutable et discutée.

D'autres propositions relatives au même thème sont apparues, dans un tout autre contexte, cette fois parmi les parlementaires de deux des principales forces partisanes du pays : le Partido Revolucionario Institucional (Parti Révolutionnaire Institutionnel, PRI) et le Partido de la Revolución Democrática (Parti de la Révolution Démocratique, PRD). En octobre 2006, le sénateur du PRI Ángel Heladio Aguirre Rivero, de l'État du Guerrero, a présenté un projet de décret afin de changer la dénomination et divers articles de la Loi (fédérale) de la Comisión Nacional para el Desarrollo de los Pueblos Indígenas (Commission Nationale pour le Développement des Populations Indigènes, CDI) et de la Ley Federal de las Entidades Paraestatales (Loi Fédérale des Entités Paraétatiques) afin d'inclure comme " ethnie » la population afromexicaine et ainsi d'inscrire sa reconnaissance dans la loi. Cette proposition s'appuie sur des sources historiques prises chez Gonzalo Aguirre Beltrán et désigne les États d'Oaxaca et du Guerrero comme étant les territoires ayant la population afromexicaine la plus importante, en la distinguant sur la base de caractéristiques phénotypiques (Diario de Debates, 2006). Deux ans plus tard, en octobre 2008, le député du PRD, Joaquín de los Santos de l'État d'Oaxaca, a proposé que l'INEGI inclue des questions dans le recensement pour savoir « si les personnes recensées sont afromexicaines, ainsi que pour établir des registres afin de déterminer leur localisation et leur nombre de manière officielle, de telle sorte que l'on soit en mesure de créer un cadre juridique les reconnaissant comme la troisième racine culturelle du Mexique et les dotant de garanties pour la préservation de leurs racines, et qu'ainsi ils existent en tant que tels dans la vie culturelle, sociale, économique et politique du pays » (SEGOB, 2008). Les deux parlementaires à l'origine de ces initiatives entretiennent des relations étroites avec les habitants de la Costa Chica sans participer pour autant aux discussions régionales sur ce thème.

On voit donc comment, ces dernières années, la revendication « afromexicaine » est devenue un thème susceptible d'être capitalisé par les institutions, les partis politiques, les candidats et les représentants populaires. Aujourd'hui, certains parlementaires - au-delà des deux mentionnés ci-dessus - incluent la population afromexicaine dans leurs discours sur la diversité culturelle. Rappelons toutefois que ces engagements ne dépassent guère le stade de discours isolés, et qu'ils n'ont pas encore de concrétisation dans l'action publique. Une tendance semble se dégager au sein des institutions gouvernementales : reconnaître les afrodescendants comme des « sujets ethniques », sans que 
cela implique un régime juridique équivalent à celui des populations indiennes, ni des politiques publiques différenciées, en dehors de celles dont ils sont bénéficiaires en tant que populations pauvres et marginalisées. On le voit, la position officielle relève encore, pour l'instant, d'un bricolage conceptuel et politique qui cherche sa cohérence.

Pour en revenir au champ de la recherche, des études ont été réalisées durant les années 1990, principalement sur des problématiques historiques et culturelles. En histoire, de nombreux travaux ont souligné l'importance démographique des populations d'origine africaine dans la société mexicaine, la diversité de leurs modalités d'insertion sociale selon les contextes productifs (mines, plantations, élevage, exploitation forestière, travaux artisanaux et domestiques) et l'hétérogénéité de leurs descendants, en particulier durant la période coloniale. Ces recherches ont restitué leurs lieux de vie et de travail, dans les espaces tant urbains que ruraux, des différentes régions du pays. De leur côté, les études anthropologiques se sont focalisées sur deux territoires, la région « jarocha» dans le sud de l'État de Veracruz, dans le Golfe du Mexique, et la région de la Costa Chica sur la Côte du Pacifique dans les États d'Oaxaca et du Guerrero9. Ces études recherchent souvent l'« empreinte africaine » dans les « traits culturels » exprimés dans la danse, la tradition orale, la musique et la gastronomie, et elles prétendent parfois démontrer ainsi une certaine continuité africaine. Certains travaux ont également incorporé dans leurs analyses les apports culturels indigènes et métis à l'actuelle " culture afrométisse », mettant l'accent sur les emprunts culturels entre populations.

Ces dernières années, plusieurs recherches ont inscrit leurs analyses dans des contextes historiques spécifiques, précisant les conditions matérielles de production et de reproduction de ces populations, ainsi que les relations de pouvoir dans lesquelles elles sont insérées. De la sorte, ces analyses remettent en question l'assimilation fréquente entre « l'identité » et « la culture » et prennent leurs distances avec les visions essentialistes de l'identité. Elles établissent des comparaisons régionales qui permettent d'identifier des formes spécifiques d'insertion sociale ainsi que le rôle des élites et de l'État dans l'élaboration des représentations et des identités collectives (Lewis, 2000 ; Alfaro et Escalona, 2002 ; Pépin Lehalleur, 2003 ; Hoffmann, 2006, 2007a, 2007b et Lara, 2007, entre autres auteurs).

Cependant, une bonne partie des recherches contemporaines persistent à étudier les afrodescendants comme un groupe séparé des populations indigènes et métisses, au moins dans la région de la Costa Chica. Elles soulignent systématiquement leurs « spécificités » et éludent la question du « métissage », considéré comme une notion marquée par l'idéologie homogénéisatrice de l'État post-révolutionnaire. Les études sur les expériences du métissage et la construction des catégories analytiques qui y sont liées restent peu développées.

Le débat anthropologique s'est développé parallèlement aux prises de position des leaders et des organisations qui interviennent dans divers forums (culturels, politiques, académiques et institutionnels), cherchant à rendre visible le thème du Noir au Mexique

9 Pour plus de références, le travail de Hoffmann (2006) fait une recension détaillée et soigneuse des études portant sur les populations afrodescendantes au Mexique. 
ou de « l'afromexicain » (expressions utilisées souvent au singulier). Ce débat tend à se polariser autour de deux positions principales, parfois caricaturées : l'une défendant « une identité noire », relative aux origines et à la diaspora africaine (Vaughn, 2004 ; Rodríguez, 2009 ) ; et l'autre affirmant une identification locale en tant que « morenos » (bronzés, bruns, foncés) relative à la couleur de peau (Meza, 2003). Ces positionnements sont cependant plus complexes qu'il n'y paraît et méritent une approche critique et sérieuse. Le thème n'est pas nouveau et des avancées conceptuelles existent, qui doivent être prises en considération pour dépasser les simplismes binaires. En effet, cette discussion se réfère aux politiques de reconnaissance de « la différence », inscrites elles-mêmes dans le cadre plus large des politiques de la citoyenneté qui orientent les processus d'inclusion et d'exclusion sociales (Rosaldo, 2000). L'enjeu dépasse donc de beaucoup un débat local entre partisans et adversaires de la reconnaissance d'un peuple noir ou d'origine africaine au Mexique.

Au total, les recherches ont évolué dans le sens d'une reconnaissance des apports des populations afrodescendantes à la construction des sociétés (locales, régionales, citadines ou rurales) dans le pays, sans qualifier ces apports comme première, seconde ou troisième racine. En même temps, elles aident à comprendre les différents rôles qu'assument les populations afros dans chacune de ces sociétés. L'analyse des dynamiques et des contextes locaux montre comment la notion de « différence » est activée à certaines échelles (locale, régionale et micro-régionale) plus qu'à d'autres, permettant ensuite de construire de nouvelles catégories analytiques. On peut ainsi distinguer des contextes où une identification régionale noire ou afromexicaine fait sens, c'est-à-dire où elle entre en résonance avec une configuration spécifique de ces territoires, des sociétés et des rapports sociaux et politiques qui s'y développent. Mes enquêtes dans la région de la Costa Chica de l'Oaxaca (Lara, 2007 et 2008) m'ont permis d'identifier des combinaisons de différentes appartenances (religieuses, de classe, de genre, partisane, de solidarité), mises en avant par les habitants « afrodescendants ». Dans certaines situations, l'identification ethnicoraciale apparaît pour disparaître ensuite, et elle peut être articulée avec des formes d'inscription dans des réseaux de relations qui correspondent à des intérêts autres qu'ethniques (bénéfices solidaires, économiques, de prestige social, etc.). Par-delà les comportements et positionnements individuels, " la différence » (surtout celle qualifiée de « raciale ») se construit aussi à partir des représentations et des discours produits par les pouvoirs publics (de l'État ou des municipalités) d'Oaxaca. Ceux-ci promeuvent et soutiennent la « reconnaissance de la différence » de façon systématique, faisant ainsi écho aux discours des collectifs revendiquant la reconnaissance d'une identité noire. Dans cette perspective, la fête constitue un mode privilégié de matérialisation du discours de la particularité ; c'est à travers elle que se met en scène « la race noire », une mise en scène qui était auparavant l'apanage exclusif des populations indigènes ${ }^{10}$.

Certains intellectuels tendent à revendiquer une « culture noire », en soulignant des spécificités qui réactualisent au passage des stéréotypes ou qui exotisent l'« identité afro » (Flores, 2006 et 2007). Ils conçoivent les collectifs « noirs » comme s'ils étaient séparés des ensembles locaux et régionaux auxquels ils appartiennent. Ils centrent la distinction sur des aspects phénotypiques interprétés comme autant d'éléments d'apparte-

10 Je me réfère à des fêtes comme la Guelaguetza, les cérémonies de la Fête Nationale ou le Carnaval. 
nance sociale et réclament leur reconnaissance en tant que groupe ethnique avec des droits associés (Rodríguez, 2009). Pour certains leaders et organisations d'afrodescendants, cette posture universitaire a l'avantage de légitimer leurs demandes face à une « communauté » plus ample (régionale, nationale), d'établir des réseaux d'alliances et de soutien à leurs demandes, et d'avoir ainsi une présence sur diverses scènes. En d'autres termes, ce type de discours académiques permet de légitimer leurs propres postures militantes. Pour d'autres leaders communautaires cependant, les catégories telles que noir, afrométis et afromexicain sont réductrices. Elles méconnaissent les multiples processus de construction identitaire, au plan local et régional, qui font référence aux liens avec les populations indigènes et métisses ainsi qu'à des identifications régionales telles que costeños (originaires de la côte), par exemple. Ces dirigeants affirment que, sans nier l'impact de l'esclavage, il est possible de nommer « la différence » sans imposer de catégories uniques, en revendiquant l'histoire " concrète », locale, et en mettant en avant la situation de pauvreté, d'exclusion et de discrimination dans laquelle vivent ces communautés.

Ces manières opposées de dire l'altérité restent l'objet de discussion entre les acteurs qui participent au débat local, montrant ainsi l'importance des analyses contextualisées qui précisent les mécanismes de production de l'altérité et reconnaissent la diversité sans l'enfermer dans des catégories univoques. D'où l'intérêt de se pencher sur le débat actuel sur la reconnaissance ethnique de la population afrodescendante dans la région de la Costa Chica, où la revendication «noire » a suscité les déclarations publiques et les mobilisations collectives les plus importantes.

\section{PROTAGONISTES DE L'ACTION ETHNO-POLITIQUE : LEADERS ET ORGANISATIONS SUR LA COSTA CHICA D'OAXACA}

\section{La position des collectivités territoriales}

On observe actuellement au Mexique des situations diverses entre les régions où l'on identifie des populations afrodescendantes, presque toujours sur la base de critères phénotypiques. Ces populations sont localisées surtout dans l'État d'Oaxaca, du Guerrero et de Veracruz et, dans une moindre proportion, dans d'autres territoires comme le Coahuila, le Tamaulipas, le Michoacán et le Guanajuato. L'histoire et les relations de pouvoir, qui nourrissent les imaginaires collectifs, ont abouti à différentes formes d'élaborations du « noir », suivant des hiérarchisations et des schémas de catégorisation raciale qui diffèrent d'un lieu à l'autre.

Dans le cas d'Oaxaca et du Guerrero, les populations afrodescendantes habitent la région de la Costa Chica sur le Pacifique Sud. Dans l'État de Veracruz, elles sont situées au sud, sur le Golfe du Mexique, dans une région que García de León (1992) a caractérisée comme la «Caribe afroandaluz » (Caraïbe afroandalouse). La perception et la connotation de la figure de l' " afro » ou du " noir » dans la région du Golfe-Veracruz et celle du Pacifique-Costa Chica sont nettement distinctes. À Veracruz, elle jouit d'une certaine valorisation positive « expliquée » par sa proximité avec la musique et la danse venues des 
Caraïbes et notamment de Cuba. L'association avec l'île promeut une image exotique, liée à la sensualité, au corps et à la beauté, aux attitudes festives, à la gastronomie, et est utilisée comme un label touristique. Dans ce contexte, la population afrodescendante ne s'identifie pas comme un groupe ethnique à part mais se perçoit intégrée à une identification régionale appelée « jarocha » qui s'assume comme métisse (indigène, noire et espagnole). A contrario, sur la Costa Chica (Guerrero-Oaxaca), les contenus associés au qualificatif « noir » sont autres et ne se fondent pas dans une hypothétique identité régionale partagée. Rappelons que les populations afrodescendantes de la Côte Pacifique étaient méconnues jusqu'à une date récente et que l'héritage culturel afro n'était pas explicité et encore moins affiché comme à Veracruz. Ces populations se concevaient plutôt comme une partie d'un ensemble régional (les " gens de la côte ») aux côtés des indigènes, majoritaires et bien visibles. Souvent même, les sources qui les montrent (reportages journalistiques ou documentaires scientifiques) présentent les groupes de populations « afro » comme des enclaves séparées de l'ensemble régional. Cependant, la valorisation de ces territoires éloignés et ruraux évolue en liaison directe avec le développement de l'offre touristique régionale et des investissements croissants - bien qu'encore très insuffisants - en infrastructures.

La reconnaissance des populations afrodescendantes par les gouvernements de Veracruz, du Guerrero et d'Oaxaca varie donc nettement d'un État à l'autre. À Veracruz, la « racine afro » est glorifiée en tant qu'elle fonde et fait partie de l'identité régionale métisse jarocha. Le gouvernement ne reconnaît pas d'identité qui scinderait ce " mélange » ou qui se différencierait comme « noire ». Et d'ailleurs il n'existe quasiment pas d'individus ou de groupes revendiquant une telle identité. Le gouvernement du Guerrero ne reconnaît pas non plus ces populations ni ne revendique une multi-culturalité. Cependant, dans cet État, trois exécutifs locaux (Cuanicuilapa, Juchitán et Copala) se sont définis récemment comme étant « des municipalités afromexicaines » et quelques collectifs locaux réclament des subventions et des programmes d'assistance spécifiques " en tant qu'afrodescendants », au niveau régional comme au niveau fédéral. Ces initiatives visent à ce que le thème de la reconnaissance des peuples noirs et des droits associés soit mis à l'agenda du gouvernement de Guerrero. À l'inverse de ces deux exemples, le gouvernement d'Oaxaca tient un discours constant depuis plus de dix ans, sur la reconnaissance multiculturelle et la revendication de la différence culturelle. Une loi régionale de 1998 reconnaît l'existence « de communautés afromexicaines » avec des droits égaux à ceux des populations indigènes. Pourtant, cette reconnaissance officielle ne précise pas comment sont constituées ces " communautés afromexicaines » et quels sont leurs membres. Elle suppose implicitement un mode de différenciation similaire à celui des populations indiennes, c'est-à-dire fondé sur une appartenance communautaire. Cette inscription des populations afromexicaines dans la loi n'a résulté ni d'une consultation ni d'une mobilisation des communautés concernées. Il ne s'agit donc pas d'un outil politique conçu, réclamé ou imposé par les acteurs politiques revendiquant aujourd'hui cette identité culturelle. À la différence d'autres cas en Amérique latine, l'accès au statut et aux prérogatives prévues par la Loi des Peuples et Communautés Indigènes de l'État d'Oaxaca ne s'appuie pas 
sur la construction d'une identité culturelle et ethnique territorialisée ${ }^{11}$ et à un système économique d'exploitation de ressources. Ce qui n'empêche d'ailleurs pas l'émergence postérieure de revendications de type ethnico-racial.

\section{Les groupes revendiquant une identité noire ou afromexicaine}

La Costa Chica est un espace pluriethnique où habitent plusieurs peuples indigènes (parlant les langues mixtèque, tlapanèque, amuzgo et chatino), ainsi qu'une population métisse et afrodescendante. On y observe un « modèle spatial racialisé » qui associe grosso modo la zone montagneuse et le piémont aux populations indigènes, les chefs-lieux municipaux et les centres urbains aux métis, les plaines du littoral et quelques villages aux «morenos » (bruns), ceux qui, aujourd'hui, commencent à s'identifier comme « afrodescendants ». Il ne s'agit cependant que de grandes tendances et, dans la réalité, on ne peut pas parler de ségrégation résidentielle des populations tant elles se mélangent.

Dans cette région, comme nous l'avons vu, la thématique noire ou afro a été impulsée en grande partie par les institutions de l'État qui sont intervenues dès les années 1980. C'est cependant l'engagement de quelques leaders qui a maintenu l'intérêt pour ce thème pendant plus de deux décennies. L'analyse des trajectoires militantes de trois de ces dirigeants est instructive car, dans cette configuration politique encore peu consolidée, ils reflètent et révèlent les grandes lignes et évolutions du débat.

Glyn Jemmoth, prêtre catholique originaire de Trinidad et Tobago, fut l'initiateur de la « question noire » sur la côte pacifique, puis son animateur. Depuis la fin des années 1980, il œuvre de façon constante pour la promotion des « peuples noirs », l'amélioration de leurs conditions de vie, et pour une prise de conscience de leur histoire et de leur identité. Les initiatives qu'il développe s'inscrivent dans la fonction de «pastorale sociale » de l'Église, mais elles répondent également aux expériences de discrimination et de racisme qu'il observe quotidiennement dans la région. Le point de départ de son engagement correspond à une volonté « de rendre conscient le rejet d'être noir » parmi les habitants de la Côte, de mettre en évidence la négation de leurs origines et de l'histoire de l'esclavage dans un pays où « la mexicanité cache la diversité culturelle » (entretien à El Ciruelo, Pinotepa Nacional, octobre 2008). Cette voie était pleine d'obstacles puisque la négation de cette identité constitue aussi une ressource stratégique pour les habitants qui cherchent ainsi à échapper à la stigmatisation et à la charge raciste associée à l'étiquette « noir ». Pour éviter cette confrontation douloureuse, le prêtre débuta son travail par la « récupération de la tradition », en particulier des danses (danse de la tortue, son de artesa), de la musique et des poésies traditionnelles toujours usitées au sein des communautés noires de la Côte. Depuis sa paroisse, le Père Glyn coordonna les initiatives culturelles et encouragea la création de l'association « México negro » (Mexique noir) avec des responsables locaux de la Costa Chica d'Oaxaca et du Guerrero, dont certains participaient également à cette époque à la fondation du Museo de las Culturas (Musée des

11 Il est important de signaler qu'une partie des villages à dominante afrodescendante se situe sur des terrains fédéraux, ce qui implique qu'ils n'ont pas de titre de propriété foncière et qu'ils peuvent être considérés comme installés illégalement, même s'ils existent depuis plusieurs décennies, voire plus d'un siècle. 
Cultures) à Cuajinicuilapa, dans l'État voisin du Guerrero. México Negro avait pour objet la reconnaissance et la fierté d' " être noir », un thème qui perdure depuis sa création. Différents projets ont été réalisés pour atteindre cet objectif central : soutien à la création artistique, projets productifs au bénéfice de l'économie familiale, projets éducatifs (installation de bibliothèques et appui à l'éducation secondaire). Cette organisation noua des alliances, principalement aux États-Unis, afin d'échanger des idées avec des militants et des universitaires et d'obtenir des financements pour ses activités. La connaissance des luttes afroaméricaines et l'expérience personnelle du Père Glyn dans les Caraïbes ont été une source d'information et d'orientation dans le travail quotidien de ce dirigeant. Ses connexions avec des réseaux d'organisations et le fait même " d'appartenir » à la communauté afrodescendante ont légitimé son rôle dans l'obtention de financements ou de soutiens à sa cause. À l'intérieur des villages, en revanche, sa légitimité à intervenir dans les problèmes communautaires repose essentiellement sur son statut religieux de prêtre.

México negro a constitué le fer de lance de la lutte pour la reconnaissance des populations afrodescendantes dans la Costa Chica et, pendant près de vingt ans (19872005), elle en a assumé le leadership. Elle a ensuite connu des tensions internes et des remises en question. Récemment, quelques membres de México Negro, paysans et cadres locaux (instituteurs, administratifs, employés), ont pris la direction de l'organisation tandis que Glyn Jemmoth fondait une autre organisation, plus petite, appelée Cimarrón, qu'il dirige. La direction actuelle de México Negro s'est réapproprié le discours de revendication de l'« afro », tout en développant de nouveaux discours et de nouvelles actions. Elle s'inspire maintenant du modèle des organisations noires latino-américaines. Cette réorientation est due en grande partie à son alliance avec une autre organisation locale, AFRICA, Alianza para el Fortalecimiento de las Regiones Indígenas y Comunidades Afromexicanas, (Alliance pour le Renforcement des Régions Indigènes et des Communautés Afromexicaines) et à un anthropologue de l'UNAM (Universidad Nacional Autónoma de México). Cette alliance s'est concrétisée par la création d'un réseau «Red de organizaciones de los pueblos negros de Oaxaca y Guerrero » (Réseau d'organisations des peuples noirs d'Oaxaca et du Guerrero) qui dénonce le racisme et le préjugé de couleur, passant ainsi d'une identification comme « afro » à « noir » ou, selon leurs propres termes, des « afromexicains aux noirs ».

La plupart des dirigeants qui militent aujourd'hui autour de la thématique de l' « afro » ou du « noir » au sein de leurs organisations respectives, ont été membres ou ont participé aux activités de México Negro. Plus récemment, des jeunes ont rejoint le réseau, apportant avec eux de nouvelles perspectives. Ils ont en particulier impulsé une utilisation d'Internet. Grâce au Web, les discussions sur " l'afro » et « le noir » parviennent maintenant davantage aux jeunes qui réélaborent ces thèmes selon leurs propres idées. De plus, Internet ouvre la possibilité d'agréger des sympathisants et des réseaux d'organisations qui adhèrent à leur cause et peuvent la soutenir à un moment donné.

Sur la côte pacifique, deux autres leaders locaux réalisent actuellement un travail intense pour la reconnaissance des « noirs » mexicains : Israel Reyes et Néstor Ruíz. Le premier préside l'organisation AFRICA, déjà mentionnée, dont le rayon d'action se limite à la Costa Chica. Israel Reyes a commencé son action en 1991, en promouvant 
la musique et la danse « afrométisses », qu'il met en exergue comme étant des éléments de la « culture noire », aux côtés de certaines croyances et pratiques rituelles. AFRICA développe aujourd'hui trois types d'alliances ou de partenariats : avec des organisations gouvernementales à caractère culturel dans l'État d'Oaxaca, avec le Programme México Nación Multicultural (Mexique Nation Multiculturelle, MNM) de l'UNAM qui gère plusieurs projets de recherche et de développement avec des communautés de la Côte et avec des organisations noires à l'étranger (Colombie, Amérique Centrale et États-Unis). Grâce à ces liens, AFRICA et son président ont réussi à établir et à diffuser la référence aux «peuples noirs d'Oaxaca » bien au-delà de la sphère locale. Le lien avec le programme universitaire de l'UNAM, en particulier, a constitué un atout important pour faire reconnaître le travail d'AFRICA dans la région et lui donner la légitimité nécessaire lors de son évolution d'un travail culturel vers un travail plus explicitement politique, comme en témoignent aujourd'hui les positions affichées sur son site Web et sur le blog associé. Les soutiens institutionnels dont bénéficie AFRICA, lui ont permis d'apparaître publiquement lors de manifestations officielles, comme le Festival Oaxaca Negra (Festival Oaxaca Noire) organisé par la ville d'Oaxaca en avril 2009, ou comme les réunions réalisées en 2008 et 2009 avec l'organisme officiel chargé des recensements de population, l'INEGI, afin de traiter la question du recensement des populations afrodescendantes.

Néstor Ruíz est un autre des leaders qui pendant ces dernières années a placé le thème « afro » au centre de son action. Il préside l'association EPOCA, Enlace de Pueblos, Organizaciones y Comunidades Autónomas (Lien entre Populations, Organisations et Communautés Autonomes). Néstor Ruíz a, depuis plus de quinze ans, une longue carrière de responsable d'organisations civiles et politiques. Son travail initial consistait à promouvoir la création d'organisations paysannes et à négocier des appuis pour les paysans. Il s'est ensuite consacré à la défense des droits de l'homme dans la région et a créé, avec une équipe de cadres et de paysans, la Comisión de los Derechos Humanos de la Costa, A.C., (Commission des Droits de l'Homme de la Costa, Association Civile). Le leadership qu'il a consolidé grâce à son travail dans ces organisations, l'a conduit à poser sa candidature à la députation locale à deux occasions. Il est élu une première fois sur la liste du Partido de la Revolución Democrática, PRD (Parti de la Révolution Démocratique, auto-qualifié « de gauche ») pour la mandature 1998-2001. À cette époque, les principales zones d'influence du PRD correspondaient aux villages indiens, alors que son implantation était très faible dans la zone dite de « la Llanada », majoritairement habitée par des populations afrodescendantes. C'est à partir de 2002 que Néstor Ruiz commence à s'intéresser aux populations de la Llanada et à s'auto-définir comme " noir ». Ce virage coïncide avec une période de crise au sein du PRD de l'État d'Oaxaca qui s'est traduite, avec le départ de plusieurs dirigeants, par des scissions aboutissant à la création de nouveaux groupes politiques s'affrontant pour définir leurs "zones d'influence » respectives. Ce contexte politique a poussé la direction d'EPOCA à focaliser ses efforts sur la Llanada et vers la population afrodescendante. Il semble donc que, dans ce cas, c'est la conjoncture politique locale qui a motivé une réorientation vers la revendication ethnico-raciale. Pour EPOCA, la population cible a changé mais elle vise toujours à obtenir puis à canaliser des ressources publiques destinées aux populations pauvres et marginalisées. Pour EPOCA, la revendication ethnique fait partie des outils de lutte pour accéder aux subventions et aux programmes sectoriels d'assistance, comme ceux qui sont destinés aux indiens. C'est la vulnérabilité sociale et économique des populations afro qui justifie la lutte pour la 
reconnaissance ethnique. Ce ne sont pas les éléments culturels qui soutiennent la revendication mais plutôt les inégalités sociales. Cependant, cette argumentation peut finir par être problématique, dans la mesure où une condition inégalitaire peut difficilement suffire à fonder une identification ethnique ; elle est cependant utilisée comme un instrument efficace de lutte sociale.

Un des outils préconisés par l'organisation dirigée par Néstor Ruiz, afin de garantir l'accès aux ressources, est la réalisation d'un recensement qui dénombre la population potentiellement bénéficiaire des programmes d'assistance, permettant ainsi de se couler dans la logique de concentration des ressources sur des « groupes cibles » marginalisés. Ces propositions diffèrent de celles des autres organisations dans la mesure où il s'agirait d'identifier des " régions noires » et non pas des individus, de sorte qu'il ne serait pas nécessaire d'enquêter auprès de la population mais de produire des évaluations chiffrées relatives à des territoires.

\section{CONCLUSION : LES CATÉGORIES DE L'ALTÉRITÉ, UN OUTIL INDISPENSABLE POUR LE RECENSEMENT?}

Curieusement, tout en partant de paradigmes et de postures relativement éloignés les uns des autres, ces trois leaders et leurs organisations s'accordent aujourd'hui pour demander un « recensement pour compter » les afrodescendants et pouvoir ainsi réclamer des droits et des programmes spécifiques.

Cette revendication d'un recensement soulève de multiples problèmes qui mettent en question sa pertinence même. Il est, par exemple, difficile d'établir l'importance démographique des populations afrodescendantes dans le pays, dans la mesure où le processus de métissage est ancien et intense depuis les premières années de la Colonie. Si l'unique paramètre était la prise en compte des traits phénotypiques, alors l'importance démographique de la population « noire » serait très réduite et très localisée. Si le critère était celui de l'auto-désignation, il est alors fort probable que très peu nombreux seraient ceux qui s'identifieraient comme étant « noirs » ou « afromexicains », d'autant que de nombreuses questions restent en suspens. Pour qui cette identité a-t-elle un sens ? Qui utilise cette catégorie et pour quoi faire ? Quelles sont les implications locales de l'usage de cette catégorie ? Son utilisation renforce-t-elle des représentations coloniales ou celles de l'élite politique ? Comment ces catégories sont-elles associées à la stratification socioéconomique et à l'appartenance à un lieu ? Que peut signifier pour la population le fait de s'identifier à une catégorie faisant allusion aux apparences et possédant historiquement une charge raciale stigmatisante?

Pour un individu, l'utilisation d'une catégorie renvoie à des notions d'appartenance, d'identification et de constitution du sujet. Pour les organisations, elle renvoie d'abord à des questions d'utilité et d'efficacité revendicative. Certains se demandent s'il ne vaudrait pas mieux développer des arguments relatifs à l'invisibilité historique, aux conditions de marginalisation sociale, économique et politique, à la discrimination et au racisme, c'est-à-dire à des questions qui ne se rapportent pas seulement aux différences culturelles. En mettant l'accent sur « la différence », et plus précisément sur sa folklorisa- 
tion par le biais de la culture exhibée, on risque fort « d'oublier » des problèmes qui sont fondamentalement politiques, économiques et sociaux. Mais inversement, ne parler qu'en termes d'inégalité suppose d'amalgamer des processus de différenciation qui ne relèvent pas tous de la distribution inégale des ressources. Il est donc nécessaire d'analyser les articulations entre les différents processus d'exclusion et d'inclusion sociale qui sont à l'œuvre selon les situations (Santos, 1995). Pour les milieux universitaires qui participent à la définition - et parfois à la construction - de cette « différence », le défi consiste alors à observer de façon détaillée les dynamiques et les contextes, afin de contribuer à la génération de catégories analytiques pertinentes.

\section{Références bibliographiques}

AGUIRRE BELTRÁN Gonzalo (1984) [1946] La población negra de México, México, Fondo de Cultura Económica, $374 \mathrm{p}$.

ALFARO Mara e ESCALONA Inti (2002) El proceso de colonización: poblamiento y formación de localidades, in Mara Alfaro y Gustavo Sánchez Coords., Chacahua: reflejos de un parque, México, CONANP-PNUD-CIESAS-SEMARNAT-Plaza y Valdés, pp. 87-112.

BILBY Kenneth (1996) Ethnogenesis inthe Guianas and Jamaica. Two maroon cases, in Jonathan D. Hill, History, power and identity. Ethnogenesis in the Americas 1492-1992, USA, University of Iowa Press, pp. 119-141.

GACETA PARLAMENTARIA Cámara de Diputados número 2617-VI, martes 21 de octubre de 2008, México, [En línea] consulta 9 de diciembre 2008. URL: http://gaceta.diputados.gob.mx/ Gaceta/60/2008/oct/20081021-VI.html

DIARIO DE LOS DEBATES, Año I, 1er período ordinario, LX Legislatura, Núm. 17, 26 de octubre de 2006, México, Cámara de senadores del Congreso de los Estados Unidos Mexicanos, pp. 28-35.

FLORES Julia (2006) Los afrodescendientes en México. Reconocimiento y propuestas para evitar la discriminación, México, Dirección General Adjunta de Estudios, Legislación y Políticas Públicas, Consejo Nacional para Prevenir la Discriminación, Colección de estudios y documentos de trabajo, $157 \mathrm{p}$.

FLORES Julia (2007) Procesos de construcción de identidad, condición de vida y discriminación en las comunidades afrodescendientes en los estados de Coahuila y Tamaulipas, México, Dirección General Adjunta de Estudios, Legislación y Políticas Públicas, Consejo Nacional para Prevenir la Discriminación, Colección de estudios y documentos de trabajo, 63 p.

GARCÍA DE LEÓN Antonio (1992) El Caribe afroandaluz: permanencia de una civilización popular, La Jornada Semanal, 12 de enero de 1992, pp. 27-33.

HILL Jonathan D. (1996) Introduction, in J. Hill, History, power and identity. Ethnogenesis in the Americas 1492-1992, USA, University of Iowa Press, pp. 1-19.

HOFFMANN Odile (2006) Negros y afromestizos en México: viejas y nuevas lecturas de un mundo olvidado, Revista Mexicana de Sociología, LXVIII (1), enero-marzo, México, Instituto de Investigaciones Sociales, Universidad Autónoma de México, pp. 103-135.

HOFFMANN Odile (2007a) Las narrativas de la diferencia étnico-racial en la Costa chica, México. Una perspectiva geográfica, in Odile Hoffmann y Teresa Rodríguez Coords., Retos de la diferencia. Los actores de la multiculturalidad entre México y Colombia, México, CEMCA, CIESAS, IRD, pp. 363-397.

HOFFMANN Odile (2007b) De las «tres razas» al mestizaje: diversidad de las representaciones colectivas acerca de lo «negro» en México (Veracruz y Costa Chica), Diario de Campo. Suplemento, (42), México, INAH, pp. 98-107. 
Informe complementario de México al comité para la eliminación de la discriminación racial (2007), [En línea] consulta 6 de octubre 2008. URL: http://www.sre.gob.mx/derechoshumanos/ docs/informes.doc

KYMLICKA Will (1996) Ciudadanía multicultural. Una teoría liberal de los derechos de las minorías, Barcelona, Paidós, 303 p.

LARA Gloria (2007) El recurso de la diferencia étnico racial en las lógicas de inclusión política. El caso Pinotepa Nacional, Oaxaca, in Odile Hoffmann y Ma. Teresa Rodríguez Coords., Los retos de la diferencia. Los actores de la multiculturalidad entre México y Colombia, CEMCA, CIESAS, IRD, ICAH, México 2007, pp. 81-110.

LARA Gloria (2008) Política, espacio y construcción social del poder local - regional en la Costa Chica de Oaxaca, Tesis de doctorado en Antropología, México Centro de Investigaciones y Estudios Superiores en Antropología Social, 339 p.

LEWIS Laura (2000) Blacks, black Indians, Afromexicans: the dynamics of race, nation, and identity in a Mexican Moreno community (Guerrero), American Ethnologist, 27, (4), USA, American Anthropological Association, pp. 898-926.

MEZA Iris (2003) "Nosotros somos morenos » etnografía de un pueblo de la Costa Chica guerrerense: Playa ventura, Tesis de Licenciatura, México, Escuela Nacional de Antropología e Historia, $123 \mathrm{p}$.

NACIONES UNIDAS (2007) Comentarios del gobierno de México sobre las observaciones finales del comité para la eliminación de la discriminación racial, 29 junio 2007, Comité para la eliminación de la discriminación racial. Informes presentados por los Estados partes de conformidad con el artículo 9 de la Convención, 22 p.

NACIONES UNIDAS, (2006) Examen de los informes presentados por los Estados partes de conformidad con el artículo 9 de la convención. Observaciones finales del comité para la eliminación de la discriminación racial: México, 4 de abril de 2006, Convención Internacional sobre la Eliminación de todas las Formas de Discriminación Racial, 5 p.

PEPIN LEHALLEUR Marielle (2003) ¿Existe el regionalismo popular? Reflexiones a partir de una región pluriétnica, in Jaime Preciado, H. Riviere d'Arc, L.A. Ramírez, M. Pepin-Lehalleur Coord., Territorios, actores y poder, regionalismos emergentes en México, México, Universidad de Guadalajara-Universidad Autónoma de Yucatán, pp. 25-48.

RODRÍGUEZ Nemesio (2009) De afromestizos a pueblos negros: hacia la construcción de un sujeto sociopolítico en la Costa Chica, in Israel Reyes et al., De Afromexicanos a Pueblo negro, México, Secretaria de Cultura del Gobierno del Estado de Oaxaca, pp. 9-17.

ROSALDO Renato (2000) La pertenencia no es un lujo: procesos de ciudadanía cultural dentro de una sociedad multicultural, Desacatos, número 3, primavera, México, CIESAS, pp. 39-49

SANTOS BOAVENTURA DE SOUSA (1995) Construçao multicultural da igualdade e da diferença, VII Congresso Brasileiro de Sociologia, Río de Janeiro, setembro, 55 p.

SECRETARÍA DE GOBERNACIÓN (2008) Con punto de acuerdo, por el que se exhorta al INEGI a incluir en los censos y conteos de población y vivienda preguntas conducentes a contabilizar a los afromexicanos, a cargo del diputado Joaquín de los Santos Molina, del grupo parlamentario del PRD, Sistema de información legislativa, México, Octubre de 2008, [En línea] consulta 1 septiembre de 2009. URL: http://sil.gobernacion.gob.mx/Archivos/Documentos/2008/12/asun 2516903_20081211_1229464380.pdf

SECRETARIA DE RELACIONES EXTERIORES (2007a) Derechos humanos: agenda internacional de México, Boletín informativo, 31 de mayo 2007, México, Dirección General de Derechos Humanos y Democracia, 2 p.

SECRETARIA DE RELACIONES EXTERIORES (2007b) Informe complementario de México al comité para la eliminación de la discriminación racial (2007), [En línea] consulta 6 de octubre 08. URL: http://www.sre.gob.mx/derechoshumanos/docs/informes.doc

TAYLOR Charles (1994 [1992]) Multiculturalisme. Différence et démocratie, trad. Denis-Armand Canal, Paris, Aubier, 144 p. 
VAUGHN Bobby (2004) Los negros, los indígenas y la diáspora. Una perspectiva etnográfica en la Costa Chica, in Ben Vinson y Bobby Vaughn, Afroméxico. El pulso de la población negra en México: Una historia recordada, olvidada y vuelta a recordar, México, Fondo de Cultura Económica/Centro de Investigación y Docencia Económicas, pp. 75-96.

Sites web consultés :

EPOCA : http://enlacedepueblosyorganizaciones.blogspot.com/

AFRICA : http://colectivoafrica.blogspot.com/

Programa México Nación Multicultural : http://www.nacionmulticultural.unam.mx/ 


\title{
La construction politique d'un sujet « afro » ou « noir » au Mexique
}

\author{
Gloria Lara
}

\begin{abstract}
Cet article porte sur l'action politique des afrodescendants au Mexique et sur l'ethnisation liée à l'identification de traits culturels et phénotypiques soi-disant « spécifiques ». Les catégories ethniques et raciales sont des constructions émanant d'acteurs universitaires, politiques, juridiques, locaux visant à reconnaître un statut particulier à la population afromexicaine. Au Mexique, on assiste à la mobilisation politique de plusieurs organisations civiles et communautaires qui revendiquent la reconnaissance constitutionnelle des «peuples noirs » et de certains droits associés ainsi que la mise en place de politiques publiques qui leur soient bénéfiques. L'article décrit l'émergence du thème « afro » dans les études universitaires et les interventions des institutions gouvernementales. Il met l'accent sur les acteurs principaux de cette action politique et sur les différentes « catégories de la différence » qui font l'objet du débat.
\end{abstract}

\section{The Political Construction of "the Afro" and "the Black" in Mexico}

\section{Gloria Lara}

The article deals with the political action of afrodescendants in Mexico and the ethnicization process related to the identification of cultural and phenotypical features considered as "specific" ones. Ethnic and racial categories are constructed by academic, governmental and legal local agents as a way to confer a specific status to Afro-Mexicans. In Mexico, the political mobilization of civil and community organizations aims at promoting the constitutional recognition of "black populations", as well as the associated rights and public policies. The analysis of the emergence of the "Afro" issue in academic studies and in governmental institutional practices put the emphasis on the protagonists of this political process and on the "categories of difference" that are at the center of the debate.

\section{La construcción política de «lo afro» y «lo negro» en México}

\section{Gloria Lara}

En este trabajo reflexiono acerca de la acción política afrodescendiente en México y el proceso de etnización sustentado en la identificación de rasgos culturales y un fenotipo supuestamente «específicos». La construcción de categorías étnicas y raciales se nutre de la producción académica, de acciones intermitentes de carácter gubernamental, de la implementación de políticas de reconocimiento multicultural en el país a partir de 1990, así como de algunos instrumentos jurídicos locales que reconocen un estatuto especial a las poblaciones afromexicanas y del contacto de éstas con los movimientos negros latinoamericanos. En México, actualmente se observa la movilización política de organizaciones civiles y comunitarias que proponen el reconocimiento constitucional de las «pueblos negros», de los derechos asociados y de políticas públicas que les beneficien. El documento describe la emergencia del tema «afro» en los estudios académicos y la intervención de instituciones del Estado, muestra quiénes son los protagonistas de la acción política y apunta las «categorías de la diferencia» que están en el debate. 